\title{
Numerical Simulation of Supersonic Flows over Ballistic Models using UST3D Programming Code
}

\author{
Arina Kryuchkova ${ }^{1,2}$ \\ ${ }^{1}$ A. Ishlinsky Institute for Problems in Mechanics, Russian Academy of Sciences, \\ Moscow, 119526, Russia \\ ${ }^{2}$ Moscow Institute of Thermal Technology, Moscow, 127273, Russia \\ kryuchkova.arina.s@gmail.com
}

\begin{abstract}
Computer modeling of a flow over two hypersonic ballistic configurations using UST3D programming code elaborated in Laboratory of Radiative Gas Dynamics of Ishlinsky Institute for Problems in Mechanics was carried out. The first configuration represented blunted cylinderflare geometry, while the second was standard HB-2 ballistic model. Simulation results showed good accordance with various experimental and numerical tests data. Non-viscous versions of UST3D code were developed to testify the numerical method validity on tetrahedral meshes. Numerical cases with the same two geometries were examined to determine solution discrepancy between non-viscous and original algorithmes. Agreement of numerical results obtained with non-viscous solver using slip boundary conditions and the original code was observed. This research demonstrated UST3D method validity for the considered range of problems.
\end{abstract}

Keywords: hypersonic flow, computer modeling, boundary conditions
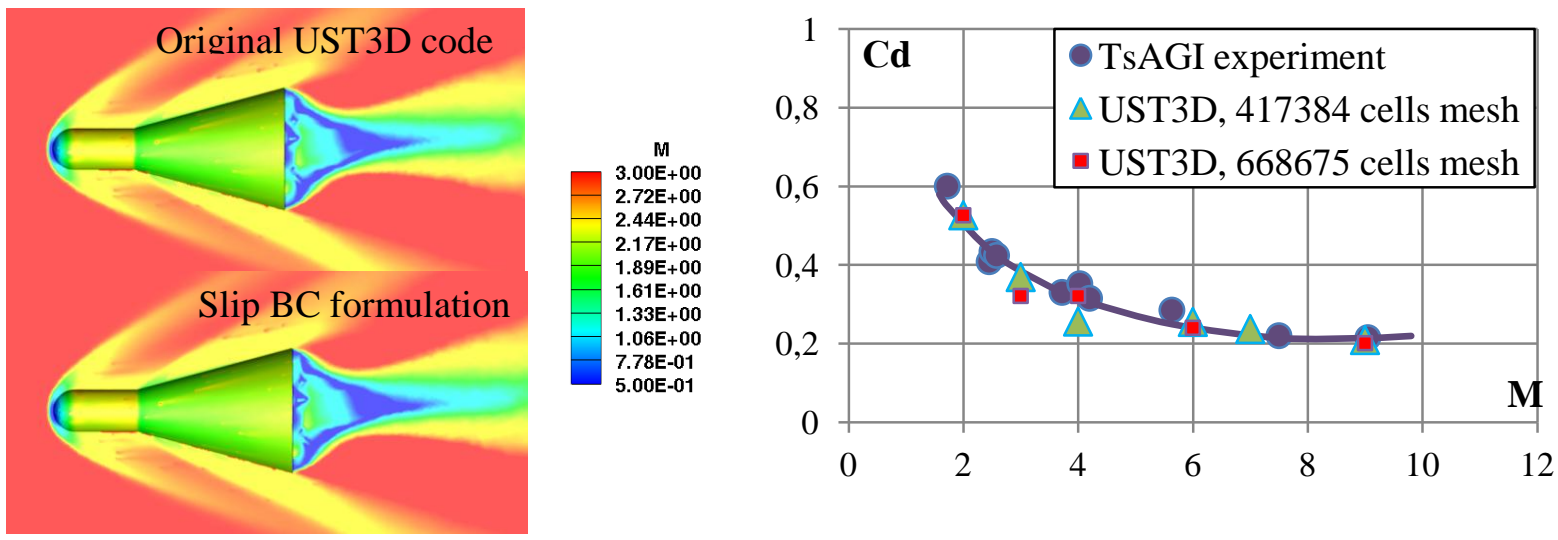

Mach number fields under $\alpha=5^{\circ}$ for blunted cylinder-flare geometry (from the left). Drag coefficient comparison for blunted cylinder-flare geometry (from the right)

Lift and drag coefficients comparison

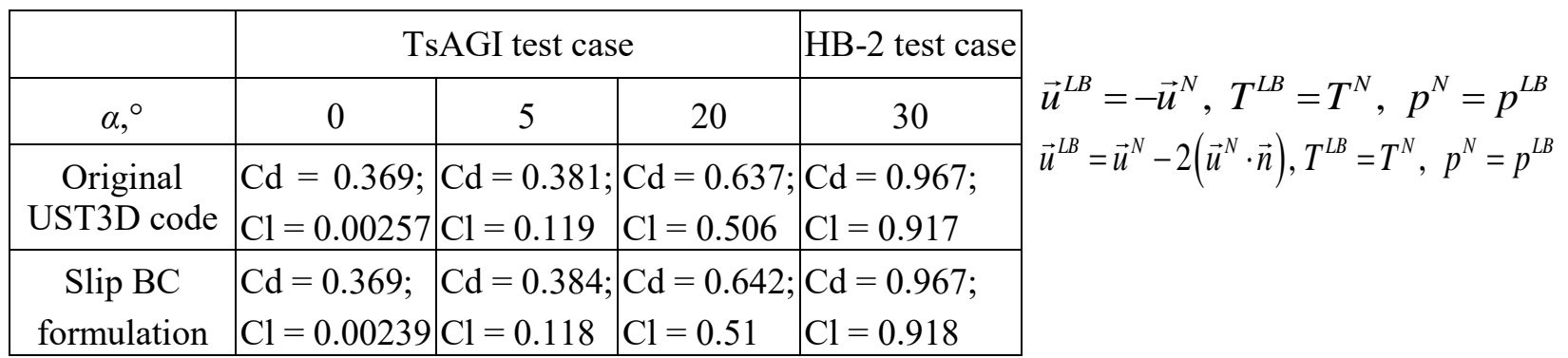




\title{
Моделирование сверхзвукового обтекания баллистических моделей в программном коде UST3D
}

\author{
А.С. Крючкова ${ }^{1,2}$ \\ ${ }^{1}$ Институт Проблем Механики им. А.Ю.Иилинского РАН, \\ Россия, Москва, 119526, пр-т Вернадского 101, к.1 \\ ${ }^{2}$ Московский Институт Теплотехники, \\ Россия, Москва, 127273, Берёзовая аллея 10, к.14 \\ kryuchkova.arina.s@gmail.com
}

\begin{abstract}
Аннотация
Программа UST3D, разработанная в лаборатории радиационной газовой динамики Института проблем механики им. А.Ю. Ишлинского, протестирована на двух задачах обтекания гиперзвуковых моделей при варьируемых углах атаки и числах Маха. Показано хорошее совпадение с экспериментальными и численными результатами других авторов. Для проверки корректности использования вязкой постановки задачи на тетраэдральных сетках разработана невязкая версия программы UST3D. Проведено моделирование нескольких расчётных случаев в невязкой постановке. Расхождение результатов, полученных в невязкой и оригинальной версиях программы, составило менее $1 \%$. Проведённое исследование свидетельствует о корректности использования программы UST3D для решения рассматриваемого типа задач.
\end{abstract}

Ключевые слова: гиперзвуковое обтекание, компьютерное моделирование, постановка граничных условий.

\section{1. Введение}

В течение последних десятилетий наиболее актуальным направлением развития аэрокосмической промышленности является освоение гиперзвуковых технологий. Первые попытки реализации гиперзвуковых программ выявили необходимость использования комбинированных методов проектирования гиперзвуковых моделей, включающих как экспериментальные, так и численные тесты [1]. Поскольку качественные наземные и летные испытания требуют больших экономических ресурсов и трудозатрат, а компьютерное моделирование становится всё более доступным, наблюдается возрастающий интерес к разработке надёжных численных методик моделирования гиперзвуковых процессов. Одними из наиболее важных показателей этих методик являются их универсальность и возможность применения в промышленности.

UST3D (Un-Structured Tetrahedral 3-Dimentional) [2, 3] - это компьютерная программа, разработанная в лаборатории радиационной газовой динамики Института проблем механики им. А.Ю. Ишлинского. Эта программа предназначена для решения широкого спектра задач газовой динамики и находится в настоящее время в стадии активного тестирования и развития. UST3D реализует численное решение пространственных уравнений Навье-Стокса посредством модифицированного метода расщепления на физические процессы $[4,5,6]$. Программа состоит из препроцессора, решателя и постпроцессора. Препроцессор собирает всю необходимую информацию о топологии вычислительных ячеек. Решатель реализует непосредственно численное решение задачи, а постпроцессор записывает результаты в подходящем для графической визуализации формате. 
На данный момент основная версия кода адаптирована для расчётов на неструктурированных тетраэдральных сетках. Выбор в пользу данного типа сеток был продиктован ориентированностью программы на применение в качестве инструмента проектирования в промышленности, что подразумевает необходимость упрощения процесса построения сетки для рядового пользователя. С другой стороны, использование тетраэдральных сеток означает, что при численном моделировании на расчётных сетках ограниченной размерности пограничный слой не разрешается должным образом. Несмотря на это, было предпочтительным сохранить вязкую формулировку кода, потому что в этом случае решение оказывается более стабильным, чем при невязкой формулировке. Кроме того, вязкая модель также может быть использована в новых версиях программы, адаптированных для расчётов на гексаэдральных сетках больших размерностей. В данном контексте особо пристальное внимание необходимо было обратить на правильность использования вязких граничных условий на стенках в актуальной версии кода.

Как известно, численное решение вязких уравнений, проводимое на сетках, не разрешающих пограничный слой, при безотрывном обтекании даёт те же результаты, что решение уравнений Эйлера. Для оценки несовпадения результатов при использовании двух постановок было предложено разработать невязкую версию программы UST3D. Расхождение результатов свидетельствовало бы о наличии паразитических вкладов от вязких членов.

Данная статья посвящена тестированию программы UST3D посредством сравнения результатов расчёта гиперзвукового обтекания баллистических моделей с экспериментом и с результатами, полученными при невязкой формулировке. В работе приводится описание реализации невязкой версии программы с двумя типами граничных условий, а также анализ сходимости решения при использовании вязкой и невязкой постановок.

\section{2. Численный метод}

\section{1. Математическая модель программы UST3D}

Как уже было сказано, UST3D реализует численное решение пространственных уравнений Навье - Стокса. В декартовой системе координат система основных уравнений может быть представлена в следующем виде:

$$
\frac{\partial U}{\partial t}+\frac{\partial E c}{\partial x}+\frac{\partial F c}{\partial y}+\frac{\partial G c}{\partial z}=\frac{\partial E v}{\partial x}+\frac{\partial F v}{\partial y}+\frac{\partial G v}{\partial z}
$$

где $U=(\rho, \rho u, \rho v, \rho w, \rho E)^{T}$ - вектор консервативных переменных;

$E c=\left(\rho u, \rho u^{2}+p, \rho u v, \rho u w, \rho u E+p u\right)^{T}, F c=\left(\rho v, \rho u v, \rho v^{2}+p, \rho v w, \rho v E+p v\right)^{T}$,

$G c=\left(\rho w, \rho u w, \rho v w, \rho w^{2}+p, \rho w E+p w\right)^{T}-$ проекции вектора конвективного потока;

$E v=\left(0, \tau_{x x}, \tau_{y x}, \tau_{z x}, u \tau_{x x}+v \tau_{y x}+w \tau_{z x}-q_{x}\right)^{T}, F v=\left(0, \tau_{x y}, \tau_{y y}, \tau_{z y}, u \tau_{x y}+v \tau_{y y}+w \tau_{z y}-q_{y}\right)^{T}$,

$G v=\left(0, \tau_{x z}, \tau_{y z}, \tau_{z z}, u \tau_{x z}+v \tau_{y z}+w \tau_{z z}-q_{z}\right)^{T}-$ проекции вектора вязкого потока;

$\rho$ - плотность; $u, v, w$ - проекции вектора скорости; $E$ - удельная полная энергия;

$\tau_{i j}$ - компоненты тензора вязких напряжений; $q_{i}$ - проекции вектора теплового потока.

Для замыкания уравнений используется модель совершенного газа

$$
p=(\gamma-1) \rho\left[E-\frac{1}{2}\left(u^{2}+v^{2}+w^{2}\right)\right],
$$

где $\gamma=1.4$ - коэффициент адиабаты воздуха. 


\section{2. Методы расщепления}

Среди широко распространённых методов отметим метод частиц в ячейках и метод крупных частиц $[4,5]$. В методе частиц в ячейках сплошная среда представляется в дискретном виде: пространство разбивается на сетку фиксированных ячеек (эйлерову сетку), внутри которых сплошная среда представляется частицами, каждая из которых несёт фиксированную массу жидкости. Далее проводится расщепление на физические процессы, которое сводится к следующим трём этапам. На первом этапе, названном авторами эйлеровым [4], моделируется изменение параметров течения в лагранжевой ячейке, совпадающей на начальном этапе с эйлеровой ячейкой, за счёт сил давления. На втором (лагранжевом) этапе моделируется движение частиц через границы эйлеровых ячеек, при этом лагранжева ячейка возвращается в своё первоначальное положение. На третьем (заключительном) этапе после возвращения лагранжевой ячейки в прежнее положение происходит пересчёт массы, импульса, и энергии на эйлеровой сетке по законам сохранения. Стационарное решение задачи получается в результате установления. Несмотря на преимущества данного метода у него существует ряд недостатков, вызванных дискретным представлением среды. Первым и самым главным недостатком является возникновение флуктуаций, особенно в областях разрежения, где располагаются ячейки с малым количеством частиц. Кроме того, из-за наличия дискретных частиц затруднено аналитическое исследование устойчивости.

Метод крупных частиц основан на тех же общих принципах, что были впервые предложены в методе частиц в ячейках. Отличие метода крупных частиц от своего предшественника заключается в замене дискретного представления сплошной среды рассмотрением совокупности непрерывных жидких частиц (крупных частиц), каждая из которых занимает в начальный момент времени весь объём эйлеровой ячейки. Таким образом, вместо совокупности частиц рассматривается масса всей ячейки в целом, а вместо перераспределения частиц по сетке изучаются непрерывные нестационарные потоки массы через эйлерову сетку. Данный подход позволил существенно увеличить запас устойчивости и устранить нефизичные флуктуации. Метод позволяет решать задачи, описывающие как дозвуковые течения, так и сверхзвуковые, включая трансзвуковые области.

Математический алгоритм метода крупных частиц можно описать следующим образом. Каждый шаг по времени состоит из описанных выше трёх этапов. На первом этапе при решении дискретного аналога уравнения (1) пренебрегают всеми конвективными эффектами. В этом случае вектора из уравнения (1) заменяются на

$$
\begin{gathered}
U^{*}=\left(\rho, \rho u^{*}, \rho v^{*}, \rho w^{*}, \rho E^{*}\right)^{T}, E c^{*}=(0, p, 0,0, p u)^{T}, F c^{*}=(0,0, p, 0, p v)^{T}, G c^{*}=(0,0,0, p, p w)^{T} \\
E v^{*}=\left(0, \tau_{x x}, \tau_{y x}, \tau_{z x}, 0\right)^{T}, F v^{*}=\left(0, \tau_{x y}, \tau_{y y}, \tau_{z y}, 0\right)^{T}, G v^{*}=\left(0, \tau_{x z}, \tau_{y z}, \tau_{z z}, 0\right)^{T}
\end{gathered}
$$

В результате определяются промежуточные значения параметров. На втором этапе на основывании вычисленных промежуточных значений скоростей определяются потоки через грани. Решение уравнения неразрывности даёт новое значение плотности в эйлеровой ячейке.

На заключительном этапе происходит окончательный пересчёт значений параметров потока по исходному виду уравнения (1), при этом используются значения промежуточных величин скорости и обновленное значение плотности.

\section{3. Реализация невязкой версии программы}

Алгоритм решателя использует принцип фиктивных ячеек на границах. Таким образом, реализация граничных условий «прилипания» имеет следующий вид:

$$
\vec{u}^{L B}=-\vec{u}^{N}, T^{L B}=T^{N}, p^{N}=p^{L B},
$$


где $\vec{u}^{N}, T^{N}$ - вектор скорости и температура в центре ячейки, прилежащей к стенке; $\vec{u}^{L B}$, $T^{L B}$ - вектор скорости и температура в центре симметричной фиктивной ячейки. На рис. 1 графически проиллюстрирован принцип постановки граничных условий с использованием фиктивных ячеек.

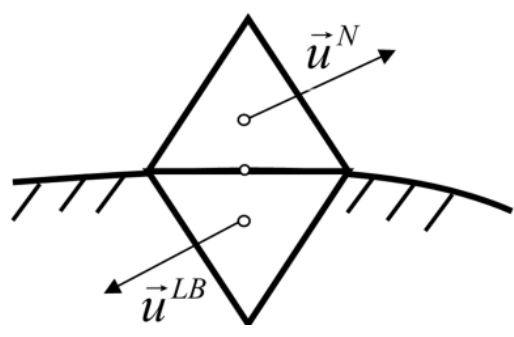

$a$

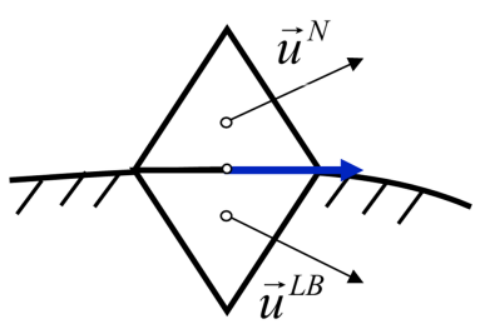

6

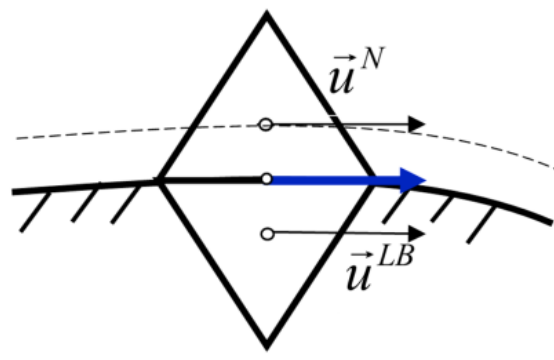

B

Рис. 1. Постановка граничных условий с использованием фиктивных ячеек: $a$ ) условие «прилипания»; б) условие «непротекания»; в) условие равенства нулю нормальных компонент вектора скорости

Невязкая формулировка достигается посредством приравнивания к нулю вязких касательных компонент $\tau_{i j}$ и постановки граничных условий «непротекания» на стенках. Последнее сводится к расстановке нулевых тепловых потоков и направления вектора скорости, касательного к поверхности. Реализация по принципу фиктивных ячеек будет иметь вид

$$
\vec{u}^{L B}=\vec{u}^{N}-2\left(\vec{u}^{N} \cdot \vec{n}\right), \quad T^{L B}=T^{N}, \quad p^{N}=p^{L B}
$$

Было также решено исследовать дополнительно вторую невязкую формулировку граничных условий. Идея заключалась в том, чтобы явным образом задать касательное направление скорости в ячейке у поверхности. Эта формулировка соответствует физическому эффекту сдвига поверхности от границы к центру прилегающей ячейки (см. рис. 1,8$)$ и позволяет оценить порядок ошибки, возникающей из-за грубости сетки. В этом случае необходимо внести поправки на значение статического давления, согласно которым обеспечивалось бы сохранение импульса в ячейке у поверхности. Численное представление подобных граничных условий выглядит следующим образом:

$$
\begin{gathered}
\vec{u}^{L B}=\vec{u}^{N^{*}}-\left(\vec{u}^{N^{*}} \cdot \vec{n}\right), \quad \vec{u}^{N}=\vec{u}^{L B}, \quad T^{L B}=T^{N}, \\
p^{L B}=\frac{p^{N^{*}}\left[2 R T^{N}+\left(\vec{u}^{N^{*}}\right)^{2}\right]}{\left[2 R T^{N}+\left(\vec{u}^{L B}\right)^{2}\right]}, \quad p^{N}=p^{L B}
\end{gathered}
$$

где $\vec{u}^{N^{*}}, p^{N^{*}}, T^{N}$ - вектор скорости, давление и температура в центре ячейки у поверхности после итерации алгоритма численного решения уравнения; $\vec{u}^{N}, p^{N}$ - вектор скорости и давление после процедуры применения граничных условий; $\mathrm{R}=287$ Дж/кг/K - газовая постоянная воздуха. Здесь и далее формулировка (5) невязких граничных условий будет обозначаться как граничное условие равенства нулю нормальной компоненты скорости (« $\mathrm{Vn}=0 »)$, а формулировка (4) - как условие «непротекания». 


\section{3. Описание тестовых примеров}

Для тестирования программы UST3D было проведено численное моделирование сверхзвукового обтекания двух типовых баллистических конфигураций. Расчётные случаи, описание которых приведено в настоящем разделе, были выбраны согласно данным экспериментов $[7,8,9]$. На тех же модельных задачах был протестирован невязкий решатель с двумя формулировками граничных условий. Все вычисления проводились на тетраэдральных неструктурированных сетках, шаг по времени выбирался автоматически из условия ограничения на невязки решения. Во всех вязких расчётах делается допущение о ламинарном характере потока. Поскольку программа адаптирована для использования на тетраэдральных сетках и, таким образом, в своей актуальной версии не позволяет проводить качественные расчёты тепловых потоков, в настоящей работе внимание уделено оценке силовых нагрузок.

\section{1. Гиперзвуковое обтекание затупленного конуса с юбкой}

Первый тестовый пример основан на данных эксперимента, который был поставлен в трубе МБУ (малая баллистическая установка) Центрального аэрогидродинамического института им. профессора Н.Е. Жуковского [7]. В этом эксперименте исследовалась аэродинамика конфигурации, состоящей из затупленного по сфере цилиндра с юбкой, обтекаемой потоком под нулевым углом атаки с числами Маха в диапазоне от 1.5 до 14. Целью эксперимента было получение структур потока, а также зависимостей аэродинамических коэффициентов при варьировании геометрических параметров модели и чисел Маха набегающего потока.

Модели затупленного цилиндра с юбкой выстреливались в рабочую часть установки со скоростями $500 \div 2000$ м/с. Числа Маха в рабочей части имели значения $2.5,3.0,3.5$. Таким образом, результирующие числа Маха составляли от 6 до 14. Для экспериментального моделирования чисел Маха от 1.5 до 6 модели выстреливались в камеру с невозмущенным воздухом. Такая методика позволила провести исследование характеристик потока в широком диапазоне чисел Маха. Числа Рейнольдса в эксперименте менялись со скоростью набегающего потока в диапазоне от $10^{6}$ до $20 \times 10^{6}$. Измерения снимались при нулевом угле атаки.

Общий вид геометрии модели представлен на рис. 2. Размеры рассмотренного в данной работе варианта конфигурации определяются следующими соотношениями: $d / 2 R=1.0$ (полусферическая головная часть); удлинение модели $L / d=1.5$; угол полураствора конуса $\theta=15^{\circ}$; полнота модели характеризовалась отношением диаметра цилиндрической части к диаметру миделевого сечения модели $d / D=0.333$. При численном моделировании диаметр миделевого сечения был принят равным 0.2 м, внешние условия соответствовали значениям $p_{\infty}=4000$ Па, $T_{\infty}=219 \mathrm{~K}$. Вычисления проводились на сетках в 417384 и 668675 ячеек, сетки сгущались вблизи поверхности модели. Расчётная область с указанием граничных условий представлена на рис. 3. Для снижения вычислительных затрат было использовано граничное условие симметрии на плоскости, совпадающей с плоскостью эскиза. 


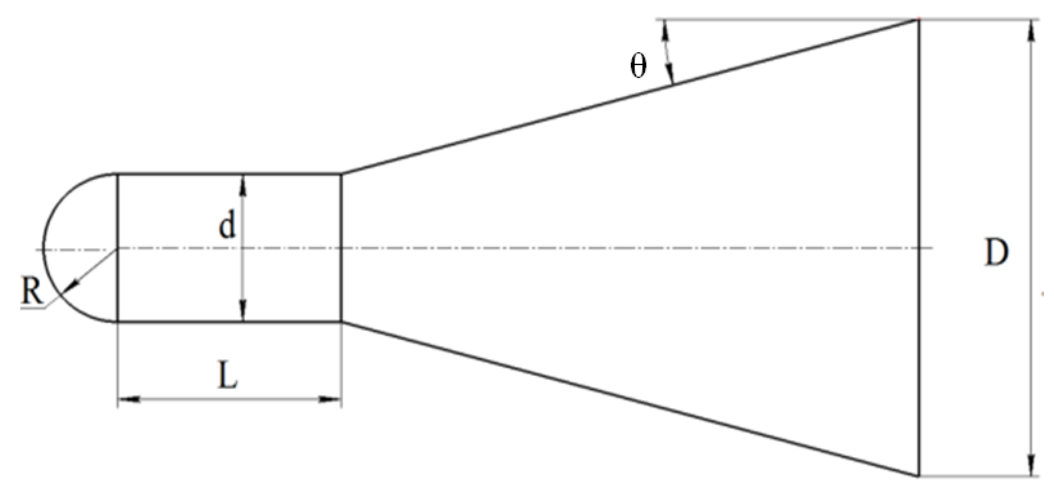

Рис. 2. Геометрия первой баллистической модели
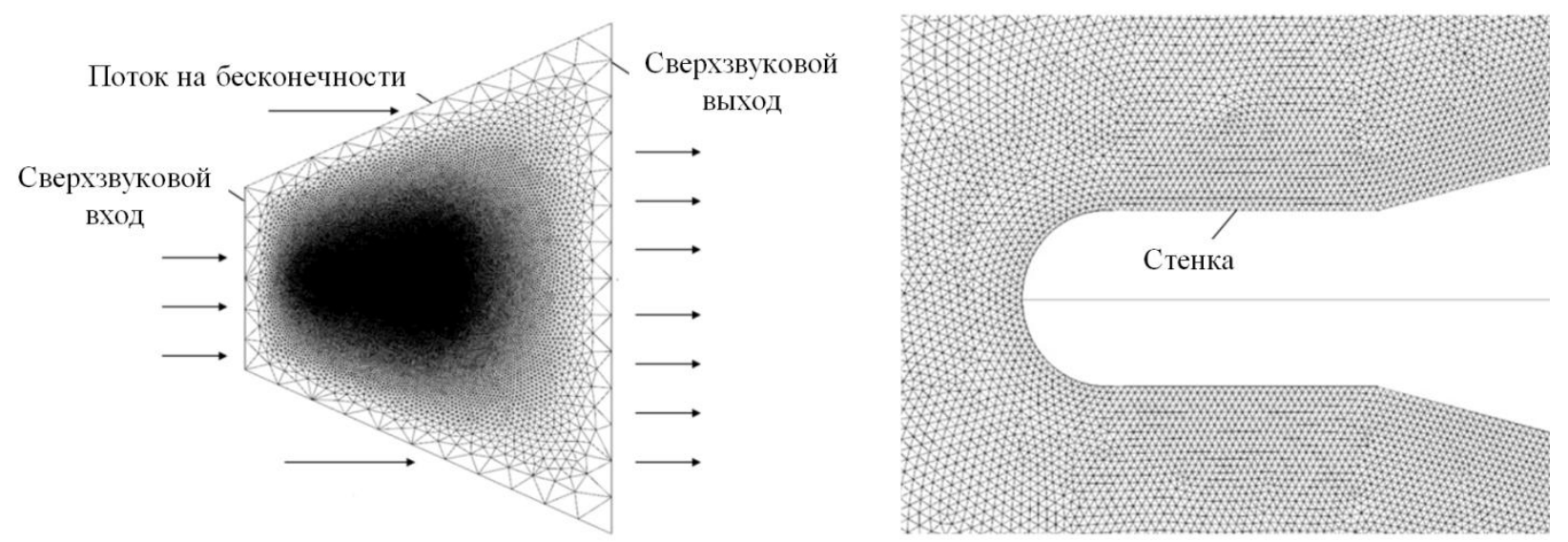

Рис. 3. Расчётная область для первой тестовой задачи с указанием граничных условий

\section{2. Гиперзвуковое обтекание баллистической модели НВ-2}

Вторая тестовая задача состояла в моделировании гиперзвукового обтекания стандартной гиперзвуковой конфигурации НВ-2. К стандартным, или по-другому, калибровочным моделям часто прибегают при проведении численных или экспериментальных исследований. Их используют как с целью проверки точности экспериментального оборудования, так и для валидации численных методов на аэродинамике хорошо изученных тел. Гиперзвуковая баллистическая модель НВ-2 представляет собой одну из двух гиперзвуковых баллистических конфигураций, предложенных Ассоциацией сверхзвуковых аэродинамических труб (STA) в 1960 году. Позднее, в 1963 модели HB-1 и НВ-2 были включены в список стандартных моделей Консультативной группы по аэрокосмическим исследованиям и разработкам (AGARD). В настоящее время эти модели широко применяются для валидации гиперзвуковых численных и экспериментальных методик [8]. Модели имеют аналитическую геометрию, которая включает затупленный конус, переходящий в цилиндр, а в случае модели НВ-2 также кормовую юбку. Геометрия модели НВ-2 изображена на рис. 4. 


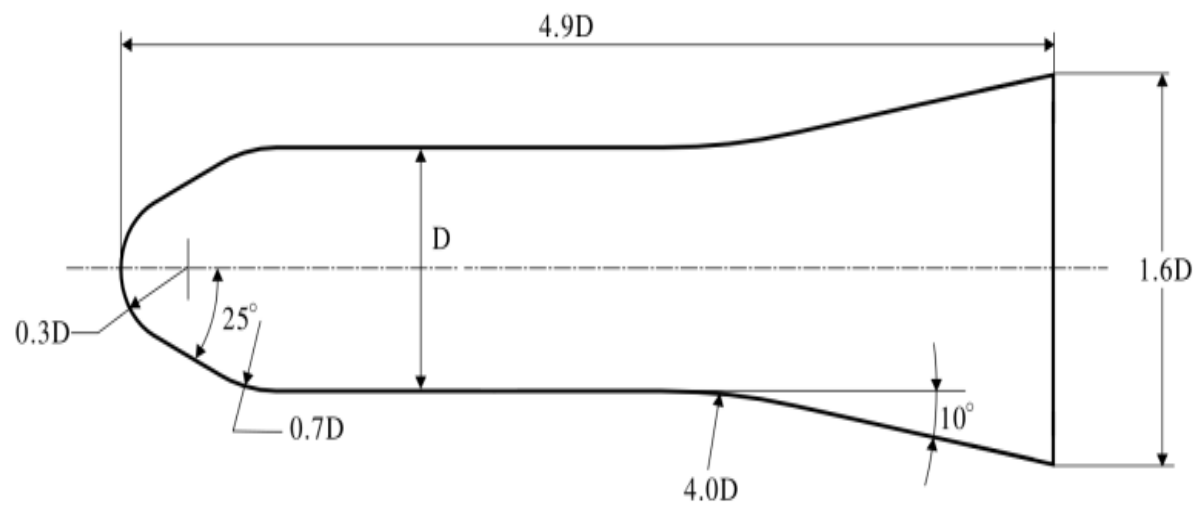

Рис. 4. Геометрия второй баллистической модели (НВ-2)

Известен ряд экспериментов по определению аэродинамики НВ-2, информацию по которым можно найти в литературе. Один из наиболее надёжных экспериментов был проведен в гиперзвуковой аэродинамической трубе (HWT) Японского агентства аэрокосмических исследований (JAXA) [9]. Данная гиперзвуковая труба относится к холодному типу и имеет номинальное число Маха, равное 10. Благодаря функциональной схеме трубы параметры потока в рабочей части могут быть измерены с высокой точностью (ошибка в оценке числа Маха менее 0.3\%). Для силовых измерений в эксперименте использовались шестикомпонентные весы (Nissho LMC-6522-33/Z100). Число Маха рассчитывалось из параметров торможения, измеряемым в камере, и по давлению в рабочей части.

В литературе также можно найти результаты экспериментов по определению характеристик модели HB-2, проводимых в трубах АT-303 (ИТПМ им. Христиановича), ARC1, R3 (ONERA,Франция), высокоскоростных аэродинамических трубах VKF (AEDC, CША), и на установках Германского аэрокосмического центра (DLR, Германия) $[10,11]$.

Кроме вышеупомянутых экспериментальных исследований было проведено достаточное количество работ, посвящённых численным исследованиям [11,12]. Результаты этих работ дополняют имеющуюся базу данных по аэродинамике модели НВ-2 и также могут быть полезными для сравнительного анализа.

В настоящей работе был проведён ряд расчётов по обтеканию модели НВ-2 при варьировании числа Маха и угла атаки. За основу был взят эксперимент ЈАХА. Внешние условия при изменении угла атаки в диапазоне от $-10^{\circ}$ до $30^{\circ}$ соответствовали случаю $\mathrm{M}_{\infty}=9.6$ , $p_{\infty}=75$ Па, $T_{\infty}=52 \mathrm{~K}$. Число Маха набегающего потока варьировалось в диапазоне от 2 до 16, внешние условия рассчитывались для каждого числа Маха по параметрам торможения, соответствующим условиям эксперимента JAXA ( $\left.p_{0}=2.5 \mathrm{MПа}, T_{0}=1000 \mathrm{~K}\right)$. Диаметр цилиндра $D$ был принят равным 1 м. При численном моделировании использовались 2 тетраэдральные неструктурированные сетки, аналогичные расчётным сеткам из первого тестового примера. Сетки насчитывали 1.4 и 3 миллиона ячеек. Их общий вид, а также применяемые граничные условия, приведены на рис. 5. 


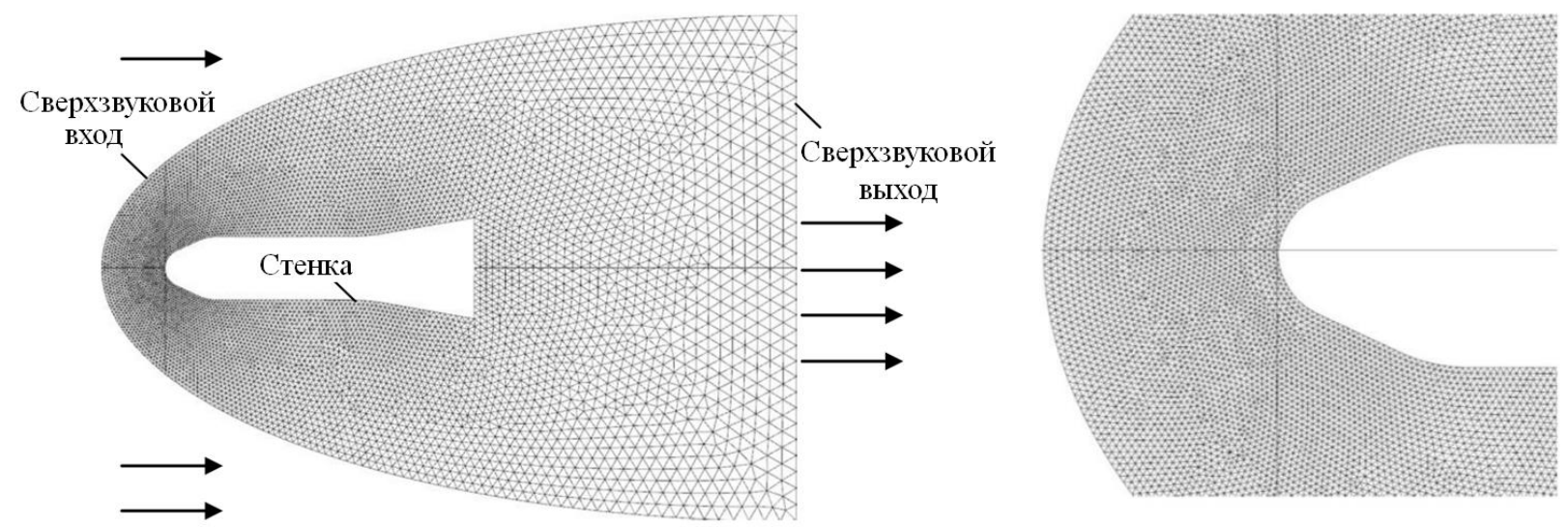

Рис. 5. Расчётная область для первой тестовой задачи с указанием граничных условий

\section{4. Результаты моделирования}

\section{1. Результаты моделирования тестовой задачи по обтеканию затупленного цилиндра с юбкой в оригинальной программе UST3D}

Структуры потока, полученные в результате моделирования в программном коде UST3D, имеют нестационарный характер, наиболее ярко выраженный в донной области. Поля числа Маха в разные моменты времени, иллюстрирующие нестационарное течение, приведены на рис. 6. Поток включает в себя такие типичные элементы, как головной скачок уплотнения, косой скачок вблизи излома образующей, и область донного течения.

Согласно расчётам с варьируемым числом Маха, для выбранной конфигурации модели отрыв течения на боковой поверхности отсутствует во всём рассматриваемом диапазоне чисел Маха, что согласуется с экспериментальными наблюдениями [7]. Сравнение полученных значений коэффициента лобового сопротивления с экспериментальными данными представлено на рис. 7. Коэффициент лобового сопротивления был отнесён к диаметру миделевого сечения модели.
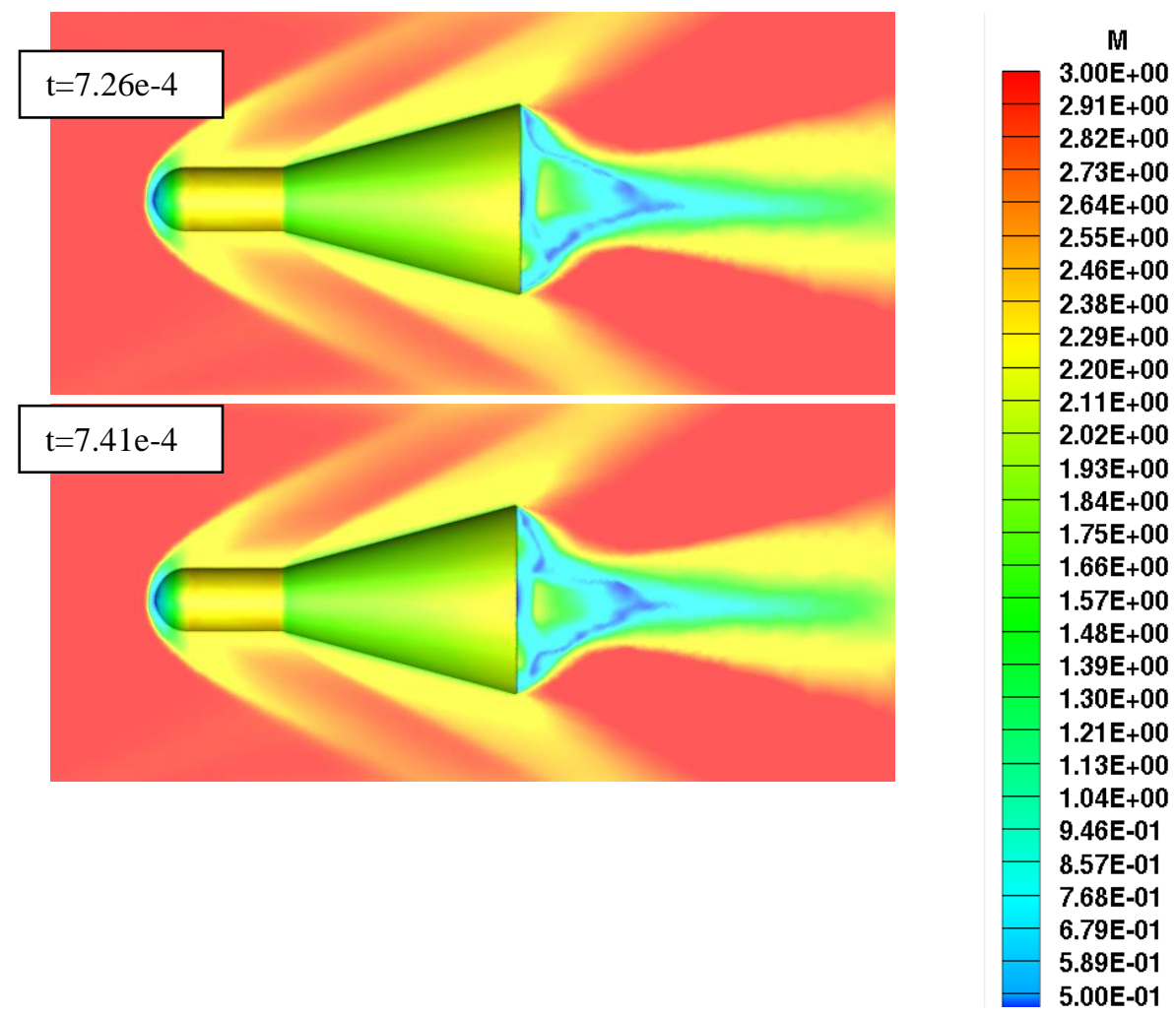


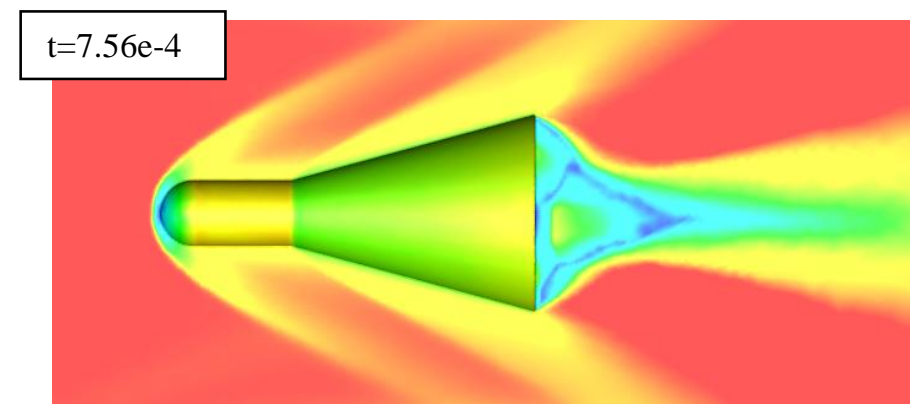

Рис. 6. Поля числа Маха для случая $\mathrm{M}_{\infty}=3$ в различные моменты времени

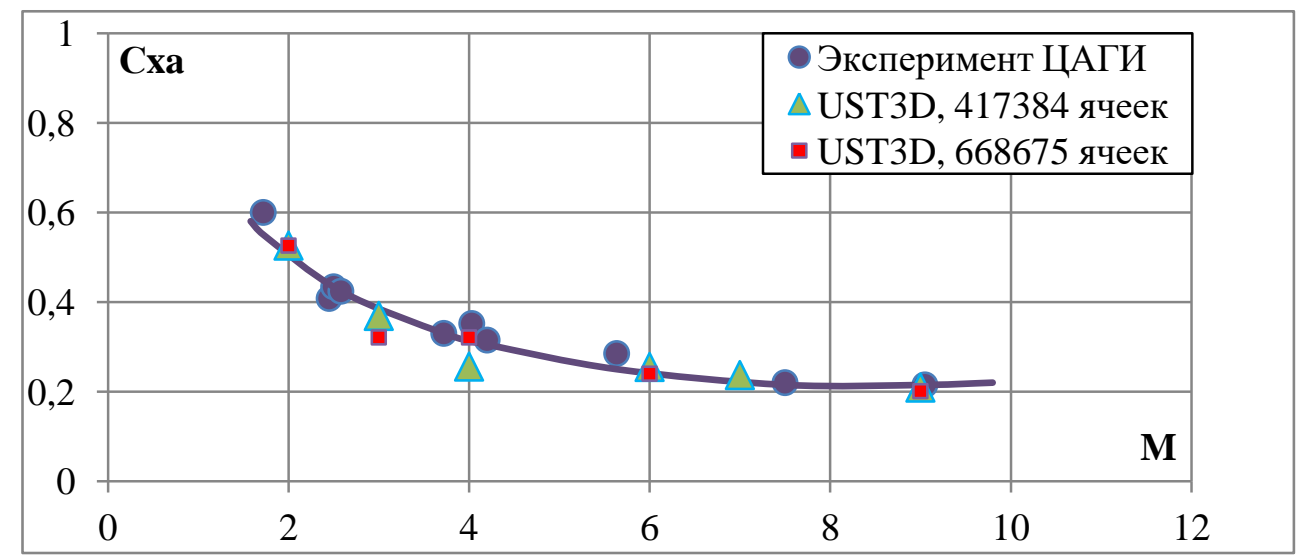

Рис. 7. Сравнение расчётных коэффициентов силы лобового сопротивления с экспериментом

Как видно из графика, сила лобового сопротивления уменьшается с ростом числа Маха и стремится к некоторому постоянному значению при достижении числа Маха, равного 7. Наблюдается хорошее совпадение численного расчёта с экспериментальными оценками. Ошибка вычислений при расчётах на обеих используемых сетках приблизительно одинакова и не превышает разброса экспериментальных значений.

Ещё одним критерием, который был рассмотрен для проверки адекватного моделирования структуры потока, являлась величина отхода головного скачка уплотнения от тела. Форма скачка и его отход в основном определяются геометрией носовой части. Поскольку носовая часть выбранной конфигурации имеет форму полусферы, отход головного скачка на оси симметрии тела может быть приблизительно оценен по известным таблицам и расчётным кривым, полученным для сферы. Существует целый ряд широко известных работ, посвящённых исследованию данного параметра [13-17]. Зависимость расстояния отхода головного скачка уплотнения от числа Маха, полученная при моделировании в UST3D, a также экспериментальные и расчётные зависимости других авторов приведены на рис. 8. Как можно убедиться, оценки, полученные в настоящей работе, хорошо согласуются с известными данными. 


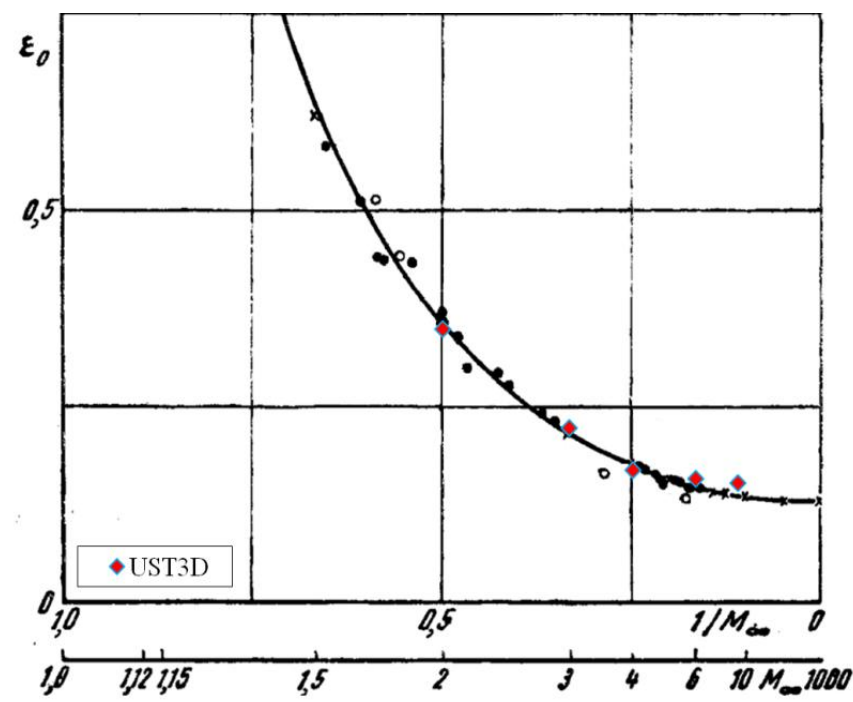

Рис. 8.Безразмерная величина отхода головного скачка уплотнения $\varepsilon_{0}=\varepsilon / R$, полученная в UST3D (красные ромбы) в сравнении с численными результатами из [14] (чёрная кривая) и [15] (кресты), а также с экспериментальными данными из [16] (белые точки) и [17] (чёрные точки)

В дополнение к расчётам с нулевым углом атаки, соответствующим условиям эксперимента, было проведено моделирование обтекания рассматриваемой модели под углами атаки $5^{\circ}, 10^{\circ}, 15^{\circ}, 20^{\circ}$ для $\mathrm{M}_{\infty}=3$. Данные расчёты послужили дополнительной проверкой программы UST3D на стабильное решение при несимметричном обтекании. Пример структуры потока, получаемой при моделировании течения под углом атаки, приведён на рис. $9, a$. Расчётная зависимость аэродинамического коэффициента лобового сопротивления от угла атаки представлена на рис. 9,6 . Согласно данной оценке, срыв потока отсутствует в пределах рассматриваемого диапазона углов атаки.

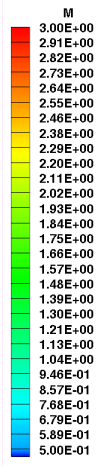

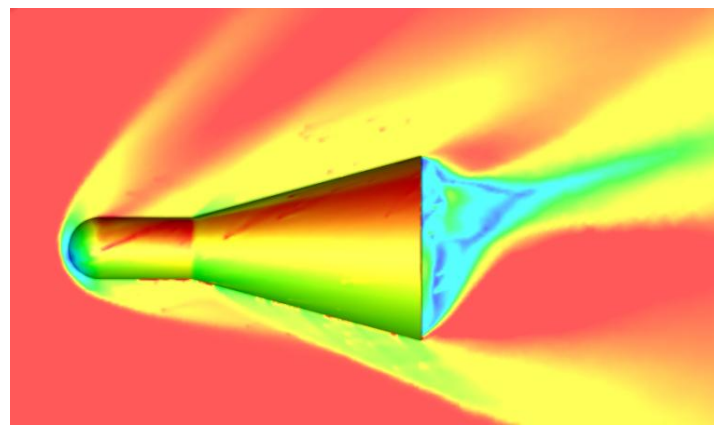

$a$

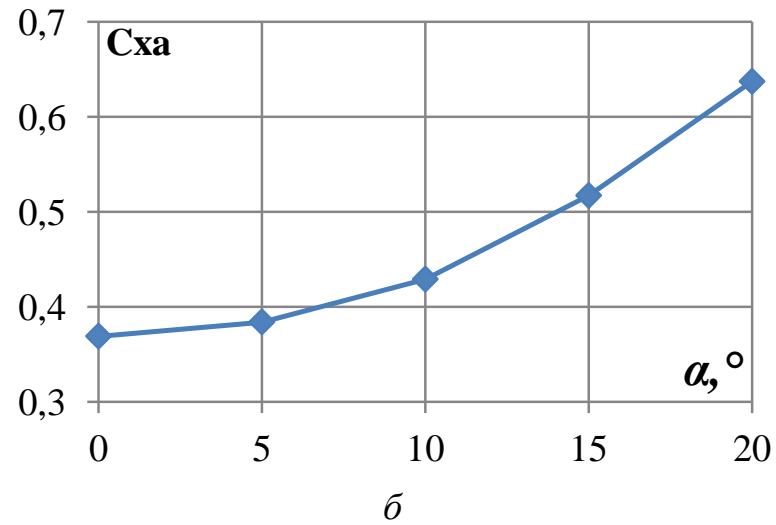

Рис. 9. Результаты моделирования потока под углом атаки: $a$ ) поле числа Маха для $\mathrm{M}_{\infty}=3, \alpha=20^{\circ}$; б) расчётная зависимость коэффициента лобового сопротивления от угла атаки для $\mathrm{M}_{\infty}=3$

\section{2. Результаты моделирования тестовой задачи по обтеканию модели НВ-2 в оригинальной программе UST3D}

При численном моделировании тестовой задачи об обтекании модели НВ-2 был получен тот же нестационарный характер течения, что и для обтекания затупленного цилиндра с юбкой. Рисунок 10 иллюстрирует изменение структуры потока, происходящее при увеличении числа Маха. На рис. 11 теневая фотография, полученная в АТ-303 для $\mathrm{M}_{\infty}=9.7$ и угла 
атаки $\alpha=12^{\circ}$, сравнена с полем числа Маха, полученным при численном моделировании потока с параметрами $\mathrm{M}_{\infty}=9.6, \alpha=10^{\circ}$.

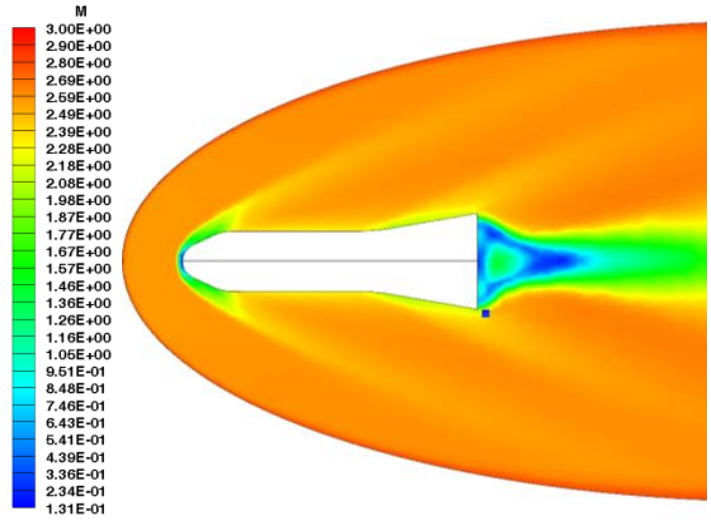

$a$
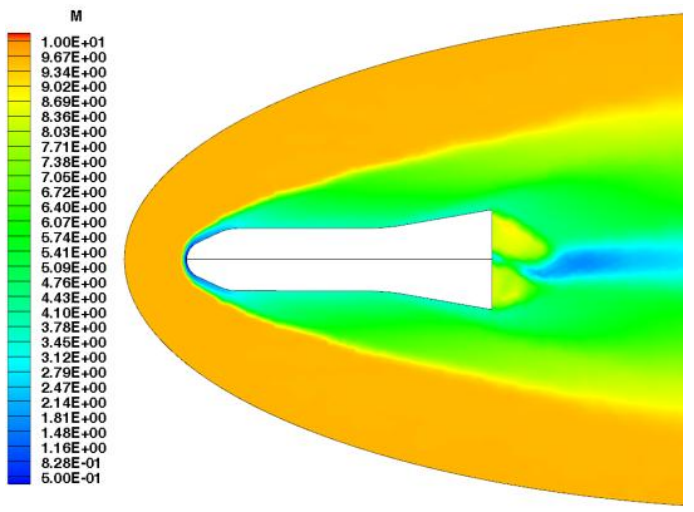

$\sigma$
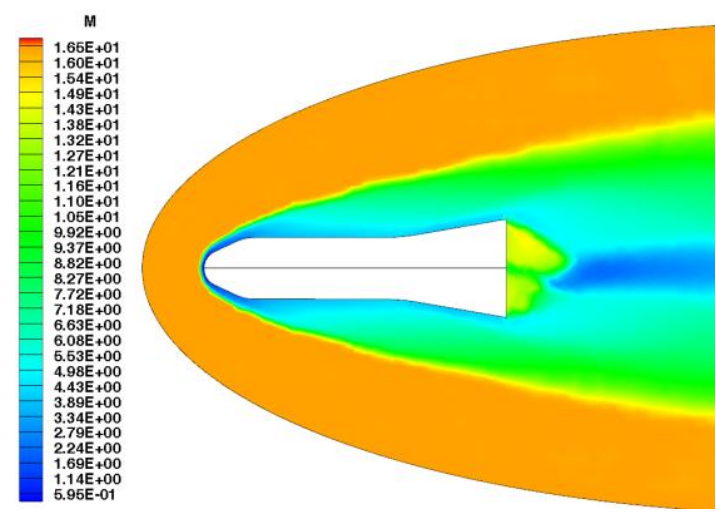

B

Рис. 10. Структура потока при различных числах Маха: a) $\mathrm{M}_{\infty}=3$; б) $\mathrm{M}_{\infty}=9.6 ;$; $) \mathrm{M}_{\infty}=16.5$

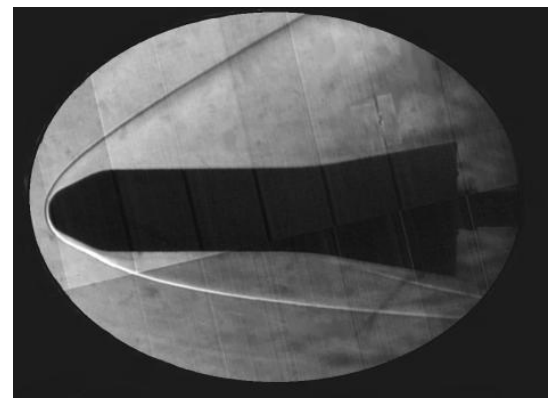

$a$

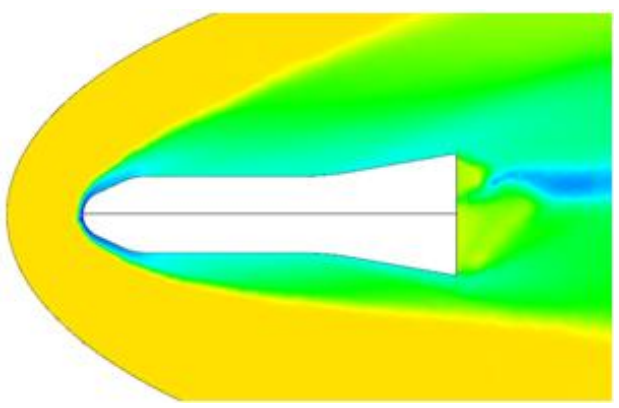

$\sigma$

Рис. 11. Сравнение структур потока: $a$ ) теневая фотография, полученная из эксперимента в АТ-303 (ИТПМ) для $\mathrm{M}_{\infty}=9.7, \alpha=12^{\circ}$; б) поле числа Маха, полученное в UST3D для $\mathrm{M}_{\infty}=9.6, \alpha=10^{\circ}$

На рис. 12 изображён график коэффициента осевой силы в зависимости от числа Маха в сравнении с экспериментальными данными из [10]. Здесь $C_{A}-$ коэффициент осевой силы с учётом донного сопротивления модели, в то время как $C_{A F}-$ коэффициент осевой силы без учёта донного сопротивления, т.е.

$$
C_{A F}=C_{A}-\frac{\left(p_{\infty}-p_{b}\right) A_{b}}{q_{\infty} A},
$$


где $p_{b}$ - донное давление; $p_{\infty}, q_{\infty}$ - давление и скоростной напор набегающего потока; $A=\pi D^{2} / 4$ - площадь сечения цилиндрической части, к которой относились значения коэффициентов; $A_{b}$ - площадь миделевого сечения. Разница в интегральных характеристиках, полученных на сетках 1.4 и 3 миллиона, оказывается менее $1 \%$ от абсолютной величины, в силу их малой визуальной различимости здесь и далее на графиках будут приведены только кривые для значений, полученных на сетке в 1.4 миллиона. Как видно из рисунка, численные результаты хорошо согласуются с экспериментом, значимое расхождение появляется только для числа Маха $\mathrm{M}_{\infty}=2$. Заштрихованная зона соответствует высоким числам Маха, для которых неточность экспериментальных измерений слишком высока и для которых могут быть обозначены только приблизительные верхние и нижние границы значений коэффициентов.

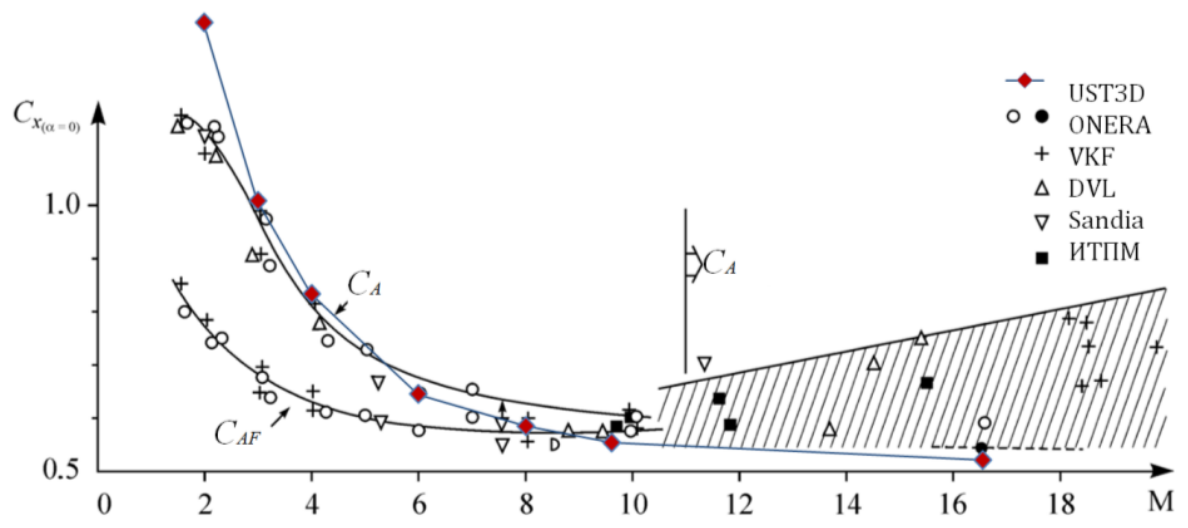

Рис. 12. Сравнение коэффициента лобового сопротивления с экспериментальными данными

Изменение структуры течения при варьировании угла атаки для числа Маха $\mathrm{M}_{\infty}=9.6$ продемонстрировано на рис. 13. Зависимости аэродинамических коэффициентов $C_{A F}, C_{N}$ осевой (без учёта донного давления) и нормальной сил от угла атаки были сопоставлены с данными эксперимента JAXA (рис. 14, 15) [9]. Максимальное расхождение между численными и эмпирическими значениями лежит в пределах $10 \%$ для $C_{A F}$ и $1 \%$ для $\mathrm{C}_{\mathrm{N}}$.

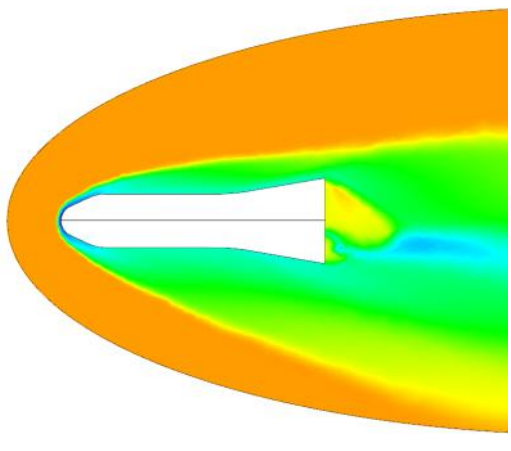

$a$

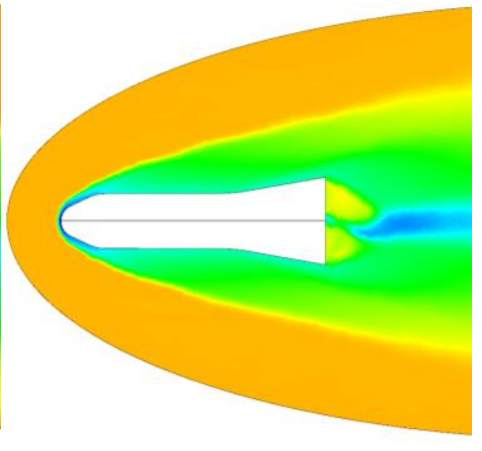

6

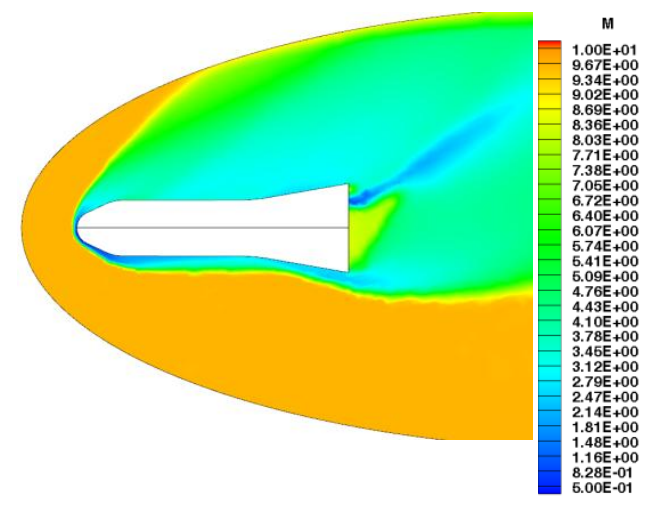

B

Рис. 13. Структура потока при различных углах атаки для случая $\left.\mathrm{M}_{\infty}=9.6: a\right) \alpha=-10^{\circ}$; б) $\alpha=0^{\circ}$; в) $\alpha=30^{\circ}$ 


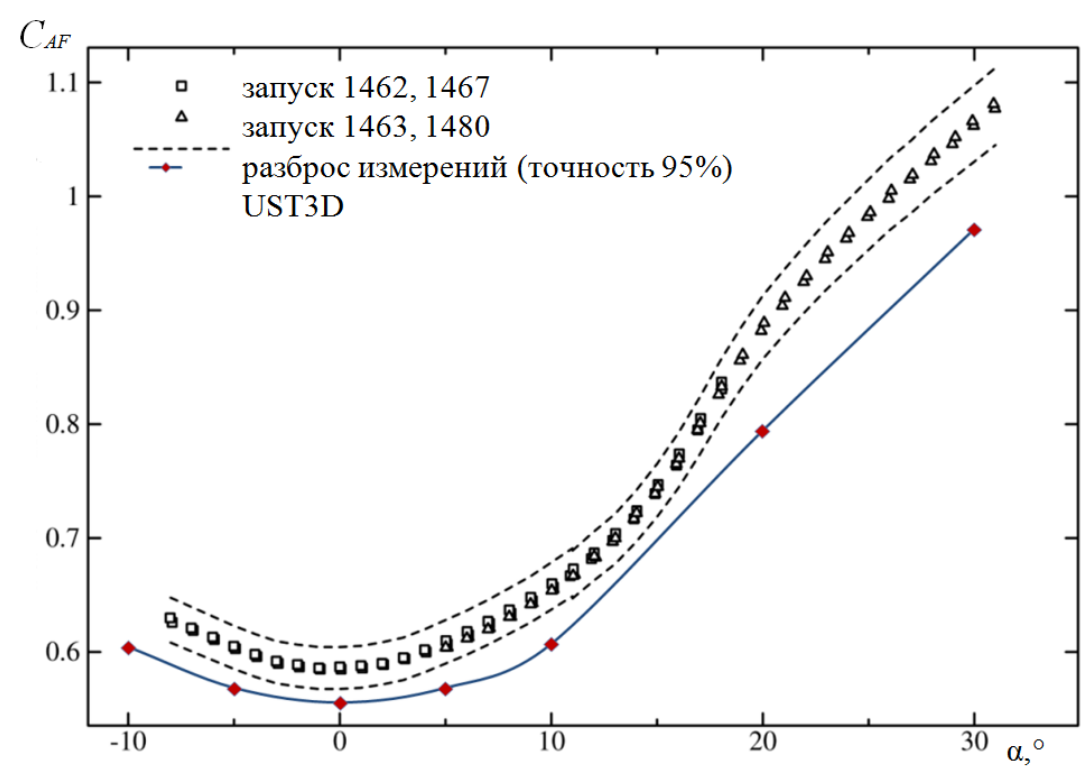

Рис. 14. Сравнение расчётной зависимости коэффициента осевой силы (без учёта донного давления) от угла атаки с экспериментом JAXA

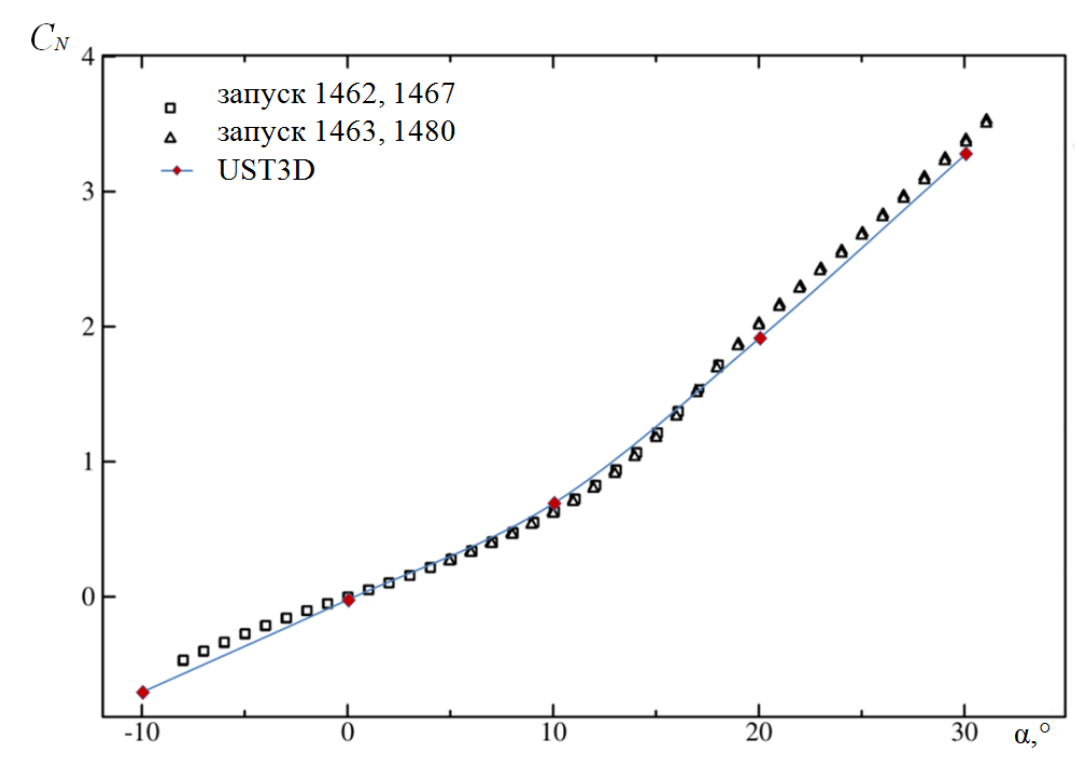

Рис. 15. Сравнение расчётной зависимости коэффициента нормальной силы от угла атаки с экспериментом JAXA

Предположение о том, что рассматриваемый гиперзвуковой поток может быть смоделирован в ламинарном приближении, нуждалось в некотором дополнительном подтверждении. Беспокойство было вызвано довольно высокими характерными числами Рейнольдса. Например, число Рейнольдса в эксперименте JAXA лежало в диапазоне от $0.9 \times 10^{6}$ до $4.3 \times 10^{6}$ на метр. Обзор литературы показал, что эффективность различных моделей турбулентности в RANS-подходе применительно к обтеканию модели HB-2 была достаточно детально изучена исследовательской группой из Белграда [11]. Группа использовала программный пакет ANSYS Fluent 16.2. Как можно судить по представленным в [11] результатам, учёт турбулентности не играет большого значения на распределение давлений (рис. 16) и, следовательно, на силовые характеристики. 
На рис. 16 показано сравнение распределения давления для $\mathrm{M}_{\infty}=4$, полученного в настоящей работе (синяя кривая), с данными из [11]. Давление отнесено к давлению торможения $p_{0}$ за головным скачком уплотнения.

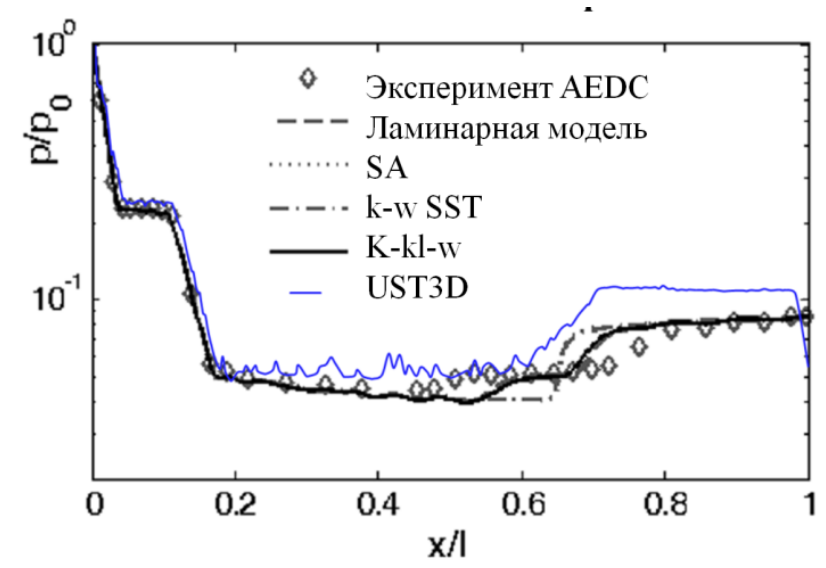

Рис. 16. Расчётное распределение давления по поверхности НВ-2 для $\mathrm{M}_{\infty}=4.0$ в сравнении с экспериментальными и численными результатами из [11]

Как видно из рисунка, кривая, соответствующая расчёту в UST3D, лежит достаточно близко к экспериментальным и численным результатам, приведённым в [11], хотя UST3D несколько завышает давление в области юбки. Это расхождение может быть связано с вычислительными ошибками, возникающими вследствие использования тетраэдральных сеток (вычисления в [11] проводились на гексаэдральной сетке).

Подобное сравнение распределения давления с экспериментальными данными JАХА и численными результатами из [12] было проведено для $\mathrm{M}_{\infty}=9.6$ (рис. 17). Может быть отмечено схожее поведение расчётной кривой, что и для $\mathrm{M}_{\infty}=4$ : значение давления несколько завышается в области юбки.

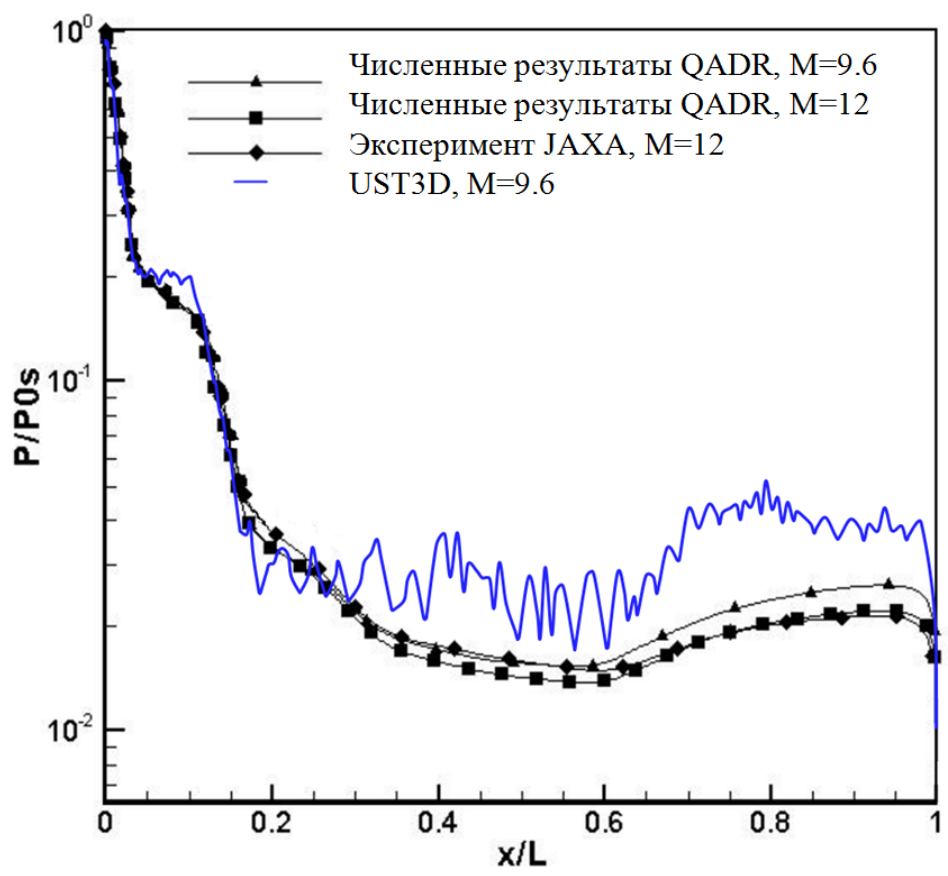

Рис. 17. Расчётное распределение давления по поверхности НB-2 в сравнении с данными эксперимента JАХА и численными результатами из [12] 


\section{3. Результаты моделирования тестовых задач в невязкой версии программы}

При тестировании невязкой версии программы UST3D также были рассмотрены две модельные задачи, описанные в разделе 3. Расчётные случаи в задаче об обтекании затупленного конуса с юбкой были ограничены значением Маха набегающего потока $\mathrm{M}_{\infty}=3$. Влияние ненулевого угла атаки было исследовано на примерах $\alpha=5^{\circ}$ и $\alpha=20^{\circ}$. В данном случае моделирование потока под углом атаки имело целью сравнение воспроизведения нормальных компонент скорости в случае использования вязкого и невязкого решателей. Моделирование обтекания модели НB-2 в невязком решателе проводилось для одного расчётного случая, соответствующего числу Маха $\mathrm{M}_{\infty}=9.6$ и углу атаки $\alpha=30^{\circ}$.

Структуры течений, полученные в невязкой версии программы с использованием граничного условия «непротекания», для всех расчётных случаев идентичны решению оригинального кода (рис. 18,19$)$. Что касается граничного условия « $\mathrm{Vn}=0 »$, то при его применении решение на поверхности становится заметно более гладким (рис. 18, в).

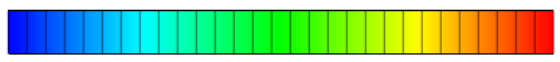

M: $\quad 5.00 \mathrm{E}-01 \quad 1.10 \mathrm{E}+001.71 \mathrm{E}+002.31 \mathrm{E}+002.91 \mathrm{E}+00$

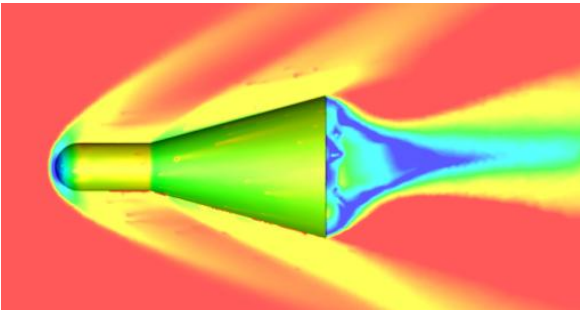

$a$

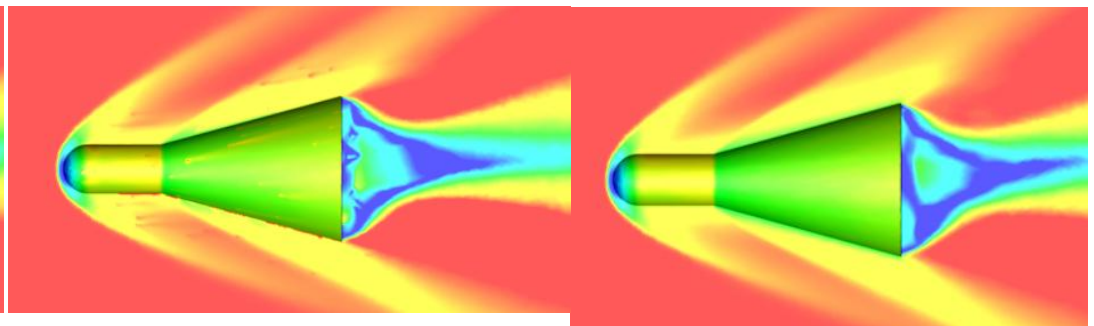

$\sigma$
B

Рис. 18. Поля числа Маха при обтекании затупленного цилиндра с юбкой для $\left.\alpha=5^{\circ}: a\right)$ с использованием оригинального UST3D; б) с использованием невязкой версии и ГУ «непротекания»; в) с использованием невязкой версии и $Г У ~ « \mathrm{Vn}=0 »$

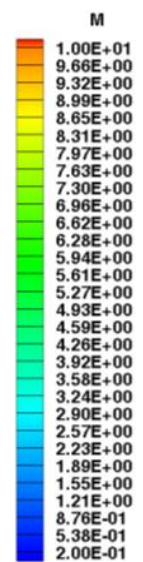

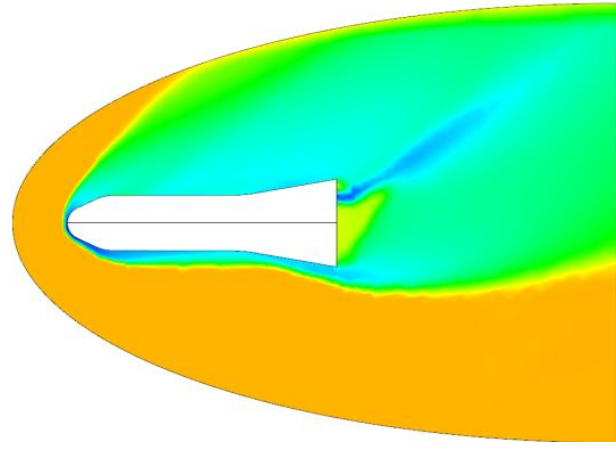

$a$

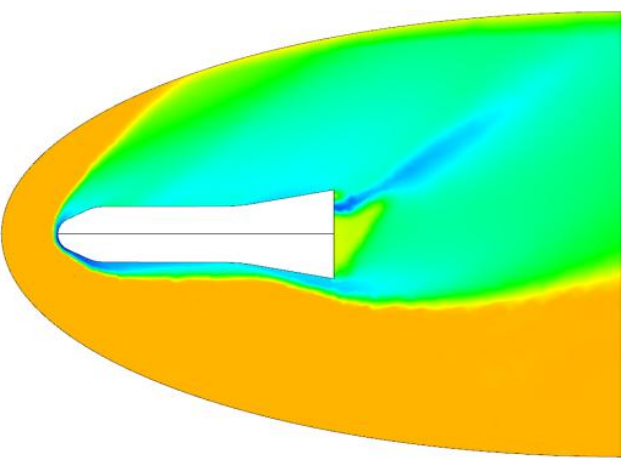

$\sigma$

Рис. 19. Поля числа Маха при обтекании HB-2 для $\left.\alpha=30^{\circ}: a\right)$ с использованием UST3D; б) с использованием невязкой версии и ГУ «непротекания»

Аэродинамические коэффициенты силы лобового сопротивления и подъёмной силы были выбраны в качестве количественного критерия, по которому производилось сравнение решений в разных версиях программы. Для обеих задач коэффициенты аэродинамических сил были отнесены к площади миделевого сечения. Результаты сравнения коэффициентов для случая обтекания затупленного конуса с юбкой сведены в табл. 1. Различие между значениями, полученными при применении условия «непротекания» и при вязкой формули- 
ровке, составляет менее $1 \%$. Силовые нагрузки на поверхность тела при использовании граничного условия « $\mathrm{Vn}=0$ » значительно ниже, разница в значениях по сравнению с результатами оригинальной версии достигает $9.7 \%$.

Моделирование обтекания HB-2 в невязкой версии UST3D проводилось только с использованием граничных условий «непротекания». Как было показано, для данного расчётного случая также сохраняется как идентичность структур обтекания, получаемых двумя алгоритмами, так и малое расхождение в оценках значений аэродинамических коэффициентов (табл. 1).

\section{Таблища 1}

\section{Сравнение значений аэродинамических коэффициентов, полученных в разных} версиях кода

\begin{tabular}{|c|c|c|c|c|}
\hline \multirow[b]{2}{*}{$\alpha,{ }^{\circ}$} & \multicolumn{3}{|c|}{ 1-я тестовая задача } & \multirow{2}{*}{$\frac{2-я \text { тестовая задача }}{30}$} \\
\hline & 0 & 5 & 20 & \\
\hline UST3D & $\begin{array}{l}\text { Сха }=0.369 ; \\
\text { Суа }=0.00257\end{array}$ & $\begin{array}{l}\text { Cxa }=0.381 \\
\text { Cya }=0.119\end{array}$ & $\begin{array}{l}\text { Cxa }=0.637 \\
\text { Cya }=0.506\end{array}$ & $\begin{array}{l}\mathrm{Cxa}=0.967 ; \\
\text { Сya }=0.917\end{array}$ \\
\hline Условие «непротекания» & $\begin{array}{l}\text { Сха }=0.369 ; \\
\text { Суа }=0.00239\end{array}$ & $\begin{array}{l}\text { Сxa }=0.384 ; \\
\text { Суa }=0.118\end{array}$ & $\begin{array}{c}\text { Cxa }=0.642 \\
\text { Суa }=0.51\end{array}$ & $\begin{array}{l}\text { Сха }=0.967 ; \\
\text { Суа }=0.918\end{array}$ \\
\hline Условие « $\mathrm{Vn}=0 »$ & $\begin{array}{l}\text { Cxa }=0.359 \\
\text { Cya }=0.00259\end{array}$ & $\begin{array}{l}\mathrm{Cxa}=0.371 ; \\
\mathrm{Cya}=0.103\end{array}$ & $\begin{array}{l}\mathrm{Cxa}=0.603 ; \\
\mathrm{Cya}=0.457\end{array}$ & - \\
\hline
\end{tabular}

Помимо сопоставления результатов решений, важной составляющей при сравнении вязкого и невязкого решателей и анализе эффективности их алгоритмов является оценка скорости сходимости. На рис. 20 приведены графики сходимости решения при использовании невязкого решателя с условием «непротекания» и оригинальной программы UST3D. Из графиков видно, что на начальном этапе решение оригинального кода сходится быстрее, хотя оба решения являются устойчивыми и достигают сходимости после приблизительно 20000 итераций. Как можно предположить, этот эффект будет более существенным при усложнении расчётной сетки.
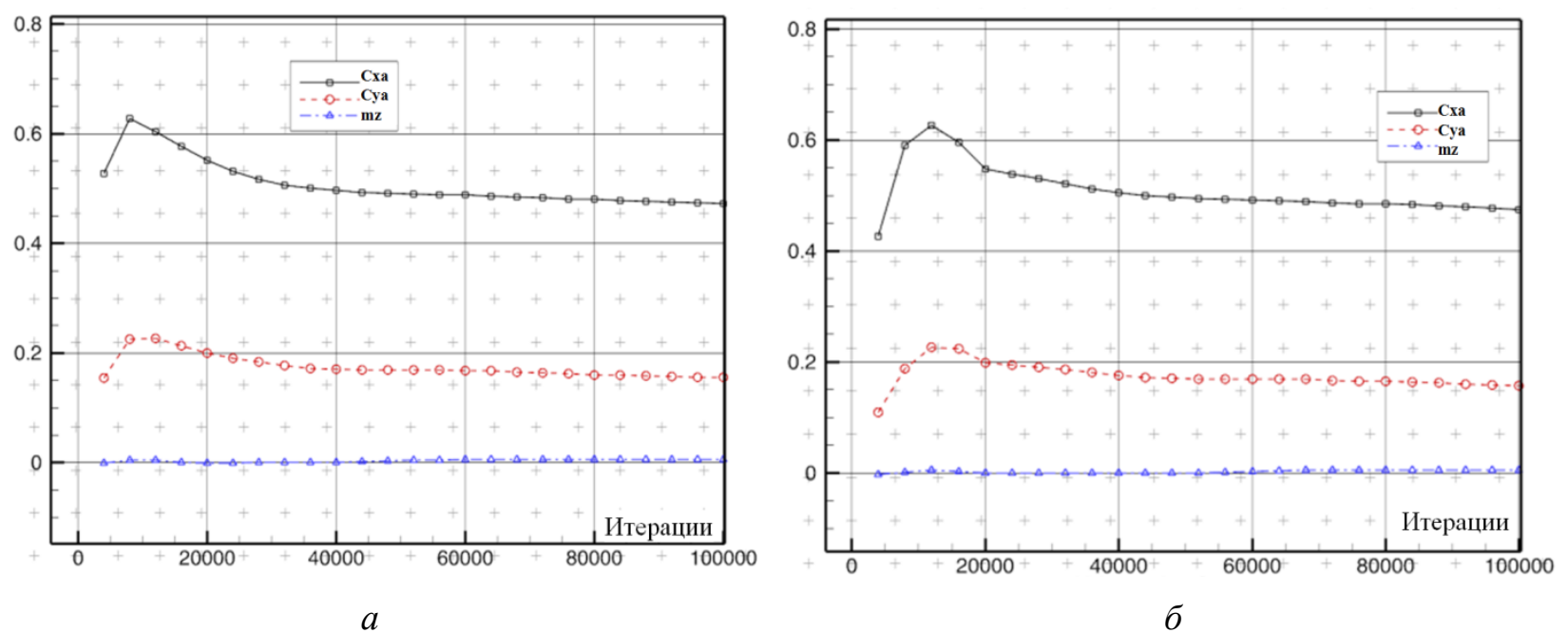

Рис. 20. Графики сходимости решения: $a$ ) с использованием оригинального кода UST3D; б) с использованием невязкой версии и ГУ «непротекания» 


\section{5. Заключение}

В работе было проведено тестирование программы UST3D на классических примерах обтекания гиперзвуковых конфигураций, представляющих собой затупленный конус с юбкой и баллистическую модель НВ-2. При численном моделировании было произведено варьирование по числам Маха и углам атаки набегающего потока. Сравнение коэффициентов лобового сопротивления с экспериментальными данными в широком диапазоне чисел Маха выявило хорошее согласование результатов вычислений с экспериментом. Расчётные зависимости аэродинамических коэффициентов от угла атаки также сходятся с эмпирическими данными с достаточной инженерной точностью. Полученные в расчёте структуры потока совпадают со структурами на экспериментальных теневых фотографиях, а также с известными данными по отходу головного скачка уплотнения. Была выявлена склонность алгоритма программы UST3D к некоторому завышению уровня давления вблизи элементов геометрии с острыми углами. Указанный недостаток, по всей вероятности, может быть исправлен путем использования более подробных или гексаэдральных сеток в случаях, когда бывает необходимо получить большую точность в оценке распределения давления.

Была реализована невязкая версии программы UST3D. Использовались два вида невязких граничных условий: условие «непротекания» и условие касательного направления скорости в ячейках у поверхности. Разработанные версии кода были протестированы на нескольких расчётных случаях рассматриваемых тестовых задач. Было продемонстрировано хорошее совпадение численных результатов, полученных при использовании оригинальной программы и её невязкой версии с граничным условием «непротекания». Использование второго вида невязких граничных условий привело к расхождению в оценке аэродинамических коэффициентов, достигающему $9.7 \%$. Такое расхождение иллюстрирует зависимость решения от качества сетки при данной формулировке граничных условий.

Идентичность результатов, полученных при использовании UST3D в вязкой и невязкой постановках, означает корректную работу программы применительно к расчётам на тетраэдральных сетках. Было также показано, что при использовании оригинальной программы шаг по времени является более гибким.

\section{Благодарности и ссылки на гранты}

Автор выражает свою благодарность профессору С.Т. Суржикову и сотрудникам лаборатории радиационной газовой динамики Института проблем механики им. А.Ю. Ишлинского РАН за оказанное содействие при подготовке настоящей статьи.

Данная работа выполнена в рамках Программы фундаментальных научных исследований РАН (контракт № АAАA-А17-117021310372-6) при частичной поддержке гранта РФФИ № 19-01-00515.

\section{Литература}

1. Kimmel R.L., Adamczak D. HIFiRE-1 Background and Lessons Learned. AIAA. 2012. 2012-1088.

2. Surzhikov S.T. Validation of computational code UST3D by the example of experimental aerodynamic data J. Phys.: Conf. Ser. 2017. Vol. 815. 012023.

3. Zheleznyakova A.L., Surzhikov S.T. Application of the Method of Splitting by Physical Processes for the Computation of a Hypersonic Flow over an Aircraft Model of Complex Configuration High Temperature. 2013. Vol. 51 (6). Pp. 816-829.

4. Ewans M.V., Harlow F.H. The particle-in-cell method for hydrodynamic calculations. Los Alamos Scientific Lab. Rept. N LA-2139. Los Alamos, 1957.

5. Белоцерковский О.М., Давыдов Ю.М. Метод крупных частиц в газовой динамике. М.: Наука, 1982. $392 \mathrm{c}$.

6. Марчук Г.И. Методы расщепления. М.: Наука,1988. 263с. 
7. Красильщиков А.П., Гурьяшкин Л.П. Экспериментальные исследования тел вращения в гиперзвуковых потоках. М.: ФИЗМАТЛИТ, 2007. 208 с.

8. Gray J.D. Summary Report on Aerodynamic Characteristics of Standard Models HB-1 and HB-2 AEDC-TDR-64-137, 1964.

9. Kuchi-Ishi S, Watanabe S, Nagai S, Tsuda S, Koyama T, Hirabayashi N, Sekine H, Hozumi K Comparative Force/Heat Flux Measurements between JAXA Hypersonic Test Facilities Using Standard Model HB-2 (Part 1: 1.27 m Hypersonic Wind Tunnel Results). JAXA Research and Development Report. JAXA-RR-04-035E. Tokyo, 2005

10. Adamov N.P., Vasenev L.G., Zvegintsev V.I., Mazhul I.I., Nalivaichenko D.G., Novikov A.V., Kharitonov A.M., and Shpak S.I. Characteristics of the AT-303 hypersonic wind tunnel. Part 2. Aerodynamics of the HB-2 reference model Thermophysics and Aeromechanics. 2006. Vol. 13 (2).

11. Pajčin M.P., Simonovic A.M., Ivanov T.D., Komarov D.M., Stupar S.N. Numerical analysis of a hypersonic turbulent and laminar flow using a commercial CFD solver. Thermal Science. 2016. Vol. 20 (6). Pp. S1-S13.

12. Mohammadifard F, Karami M, Heidari M. Investigation of Flow Field in a Typical Hypersonic Wind Tunnel over a Standard Mode. J. of Applied Fluid Mechanics. 2013. Vol. 6 (4). Pp. 529-536.

13. Krasil'shchikov A.P. and Podobin V.P. Experimental study of sphere aerodynamic characteristics in free flight up to M 15 Fluid Dynamics. 1968. Vol. 3 (4). Pp. 132-134.

14. Базжин А.П., Благосклонов В.И., Минайлос А.Н., Пирогова С.В. Обтекание сферы сверхзвуковым потоком совершенного газа// Учёные записки ЦАГИ. 1971. Т. 2. №3. С.95-101.

15. Любимов А.Н., Русанов В.В. Течение газа около тупых тел. М.: Наука, 1970. 668c.

16. Липман Г.В., Рошко А. Элементы газовой динамики. М.: Изд-во иностр. лит., 1960. 352c.

17. Масленников В.Г. Исследование положения отошедшей ударной волны при сверхзвуковом движении эллипсоидов вращения в газах с различной внутримолекулярной структурой// Сборник «Аэрофизические исследования сверхзвуковых течений». М. - Л: Наука, 1967. 241с. 